\title{
A Criterion for Homogeneous Linear Differential Equations with Damped Solutions
}

\section{AUREL WINTNER}

\author{
Communicated by L. CESARI
}

1. The results of this paper center about the following Abelian lemma:

(i) If $\omega=\omega(r)$, where $0<r \leqq r_{0}$, is any continuous function for which the product $r \omega(r)$ stays between two positive bounds as $r \rightarrow 0$, then there exists a constant $\alpha=\alpha(\omega)>0$ having the property that

$$
x(r)=O\left(r^{\alpha}\right) \text { as } r \rightarrow 0
$$

(and so, in particular, $x(+0)=0$ ) holds for every solution $x(r)$ of the differential equation

$$
\frac{d^{2} x}{d r^{2}}+\omega^{2} x=0
$$

If in addition lim inf $r \omega(r)>\frac{1}{2}$, then, on the one hand, (1) holds for $\alpha=\frac{1}{2}$ (no matter what the value of $\lim \sup r \omega(r)<\infty$ may be) and, on the other hand, (2) is stable in the sense of being oscillatory (that is, every real-valued solution $x(r) \neq 0$ of (2) must change sign an infinity of times as $r \rightarrow 0$ ).

The last assertion (but nothing like the estimate (1) or, for that matter, just $x(+0)=0$ ) is clear from STURM's comparison theorem. For, according to that theorem, it is sufficient to ascertain the oscillatory character of (1) when $r \omega=r \omega(r)$ is a constant $\omega_{0}>\frac{1}{2}$. But a direct substitution shows that, if $r \omega(r)=\omega_{0}$, then $x(r)=r^{\lambda}$ is a solution of (2) if $\lambda^{2}-\lambda+\omega_{0}^{2}=0$. Since the roots of this quadratic equation are $\lambda=\frac{1}{2} \pm i \beta$, where $\beta>0$, if $\omega_{0}>\frac{1}{2}$, the assertion follows from the fact that the zeros of the cosine or of the sine of $\beta \log r$ cluster at $r=+0$.

A (formal) logarithmic differentiation of the $O$-estimate of (i), or of the corresponding $O$-estimate in (ii) below, shows that the issues involved are related to PoIncaré's problem concerning Riccati's equation (cf., e.g., [2], pp. 53-54). Correspondingly, the $O$-assertions in question are of the same type as in classical considerations of LiAPounoff [6], pp. 223-224, and PERron [7], [8]. But there the assumptions are quite different and, mainly because of the inclusion of the oscillatory case, quite a different approach will have to be 
followed in the proof of (i) or (ii) below. In fact, what would be needed for a LIAPOUNOFF-PERRON type of approach to the problem of a linear differential equation of order $n$ is a (unilateral) comparison theorem for the set of $n$ indices which are the order numbers in the terminology of Perron [8]. Such a general approach will be developed elsewhere.

The method to be used will succeed by having recourse to a theorem proved in [12], a theorem the scale of which is finer than the logarithmic scale of RICCATI's equation. But it will be clear from the proof that, at the cost of more elaborate estimates on the rougher scale, the finer result of [12] could be avoided even in the proof of the main result, which is (ii* bis) at the end of the paper.

The non-trivial part of (i), represented by (1), where $\alpha>0$, can be extended from (i) to the general "Fuchsian" type

$$
r^{2} \frac{d^{2} x}{d r^{2}}+\psi(r) r \frac{d x}{d r}+\varphi(r) x=0
$$

where both coefficient functions $\psi(r), \varphi(r)$ are bounded, and $\varphi(r)$ has a positive lower bound, as $r \rightarrow+0$. Under entirely different conditions (conditions which amount to

$$
\int_{+0}|\psi(r)| d r<\infty \text { and } \int_{+0}|\varphi(r)| d r<\infty
$$

except in a degenerate "parabolic" case, a case of multiple elementary divisors, for which more than (4) is needed), the asymptotic behavior of the solutions of (3) was determined by Bôcher [1]. But if no integral condition (of the type

$$
\int_{+0}|\chi(r)| d r<\infty \quad \text { or } \quad \int_{+0}|d \chi(r)|<\infty,
$$

where $\chi=\psi, \varphi$; cf. [9]) is imposed, then, in view of known examples, one might expect that not even $x(r)=O(1)$, as $r \rightarrow 0$, will in general hold for the solutions of (3). But it turns out that the qualitative Tauberian restriction lim inf $\varphi(r)>0$ prevents $x(r) \neq O(1)$, and more, if, instead of (4), it is assumed that $\varphi(r)=O(1)$ and $\psi(r)=O(1)$ :

(i*) If $\varphi(r)$ and $\psi(r)$, where $0<r \leqq r_{0}$, are real-valued, continuous functions which are bounded as $r \rightarrow 0$, and if $\varphi(r)$ stays above a positive bound and $\psi(r)$ stays below a bound which is less than 1 , then there exists a constant $\alpha=\alpha(\psi, \varphi)>0$ having the property that (1) (hence, in particular, $x(+0)=0$ ) holds for every solution $x(r)$ of (3).

Clearly, (i) is a particular case $(\psi \equiv 0)$ of $\left(i^{*}\right)$. But it turns out that (i*) can be concluded from (i).

2. What will be involved in the proof of (i) or (i*) is a peculiar extension of LAGRANGE's principle of the variation of constants. For, on the one hand, the essential point in (i) is that the oscillatory case is not excluded ( $c f$. the corre- 
sponding circumstance emphasized after the definition of the "(*)-type," introduced, for $t \rightarrow \infty$, below) and, on the other hand, the traditional form of LAGRANGE's device applies to non-oscillatory differential equations only (in this regard, $c f$. [5], pp. 635-636).

The extension of LAGRANGE's principle to the non-oscillatory case has, besides (i) (or, equivalently, a main result of this paper, which is the result (ii) below) other applications also. The situation is the more curious as everything will be reduced to a theorem proved in [12], a theorem which, in the scalar case (A. KNESER; cf., e.g., [2], pp. 46-54), is very much of a non-oscillatory type.

A systematic development of the implications of the resulting extended, possibly oscillatory, form of the principle of the variation of constants, as a method of estimation by comparison, will be given elsewhere. In what follows, (i) or (i*) will be dealt with independently, partly because the main points are not encumbered by secondary complications in this case, and partly because what thus results contains an explicit answer to an "applied" question of classical type; $c f$. (ii) below.

3. Let a real- or complex-valued function, defined for large positive $t$, be called exponentially small or, for the sake of brevity, a $\left(^{*}\right)$-function, if it is $O\left(e^{-\gamma t}\right)$ as $t \rightarrow \infty$, where $\gamma$ is some (sufficiently small) positive constant. The real subclass of these functions is more inclusive than is the class of functions which, in the customary nomenclature of the applications, have graphs representing "decay." In fact, not only $t^{\star N} e^{-\gamma t}$ but also $e^{-\gamma t} \cos t$ is a (*)-function.

Let an ordinary differential equation (which will always be assumed to be linear and, for the sake of simplicity, homogeneous, with coefficients which are given as continuous functions for large positive $t$ ) be called of type $\left(^{*}\right)$ if all of its solutions are $\left(^{*}\right)$-functions. For instance, the trivial differential equation $p(D) x=0$, where $D$ denotes $d / d t$ and $p=p_{n}(\lambda)$ is a polynomial $a_{0} \lambda^{n}+a_{1} \lambda^{n-1}+$ $\cdots+a_{n}$ (with $n>0$ and $a_{0} \neq 0$ or $a_{0}=1$ ), is of type $\left(^{*}\right)$ if and only if each of the (not necessarily distinct) roots of $p_{n}(\lambda)=0$ has a negative real part. If all $n+1$ coefficients $a_{k}$ of $p_{n}$ are real, this will be the case if and only if

$$
\left|\begin{array}{lllll}
a_{1} & a_{3} & a_{5} & \cdots & a_{2 m-1} \\
a_{0} & a_{2} & a_{4} & \cdots & a_{2 m-2} \\
0 & a_{1} & a_{3} & \cdots & a_{2 m-3} \\
0 & a_{2} & a_{4} & \cdots & a_{2 m-4} \\
\vdots & & & & \vdots \\
. & \cdots & & & a_{m}
\end{array}\right|>0 ; \quad m=1,2, \cdots, n,
$$

where $a_{h}$ is meant to signify 0 if either $h<0$ or $h>n$. This criterion, which avoids the labor of calculating the roots of $p_{n}(\lambda)=0$, was discovered, independently, by RouTH and HuRwitz (in connection with two hardly different questions 
in applied mathematics). A proof of this criterion can be found in some texts of algebra (e.g., [3], pp. 235-241; cf. also pp. 159-162 of Grommen's Göttingen thesis [4], suggested by ToEpLiTz, where the criterion is extended from polynomials $p_{n}$ to certain transcendental entire functions $p_{\infty}$ ).

4. If $n=2$, and, without loss of generality, $a_{0}>0$, then it is readily seen that the determinant inequalities reduce to $a_{i}>0$ for $j=1,2$, and so (if $a_{2}$, $a_{1}, a_{0}$ are denoted by $f, g, 1$ respectively) the case $n=2$ of the RourH-HuRwitz criterion is the following trivial fact: If $g$ and $f$ are real constants, then the pair of conditions

$$
g>0, \quad f>0
$$

is necessary and sufficient in order that the real parts of both roots of $\lambda^{2}+g \lambda+$ $f=0$ be negative; hence, in order that

$$
x^{\prime \prime}+g x^{\prime}+f x=0,
$$

where the primes denote $d / d t$, be of type $\left(^{*}\right)$. The following considerations deal with the problem of the $\left({ }^{*}\right)$-character of (6) when the coefficients, instead of being constants, are arbitrary (real-valued) continuous functions $g=g(t)$, $f=f(t)$ of $t(\rightarrow \infty)$.

The main point will be the inclusion of the stable (rather than just of the "decay," or strictly "damped") case of a non-stationary equation (6) of type $(*)$, that is, the inclusion of the oscillatory case. The interpretation of any oscillatory case of (6) as a case of certain stability was introduced by the Treatise of THOMson and TAIT on the basis of a dynamical argument (expressible in terms of the long-range behavior of a regulator mechanism) but, as pointed out in [13], this interpretation is consistent with, though of course it does not imply, the strict stability of the case $g \equiv 0$ of $(6)$; cf. also [14]. In the stationary case ( $g=$ const., $f=$ Const.) of (6), the oscillatory character is defined by the condition $\beta \neq 0$ for the roots $\lambda=\alpha+i \beta$ of the characteristic equation (even if (6) fails to be of type $\left(^{*}\right)$, that is, even if $\alpha \geqq 0$ ). In the general case, the need for a criterion for the $\left(^{*}\right)$-character of the nonstationary differential equation (6) arises, of course, from the circumstance that an "explicit integration" of (6) almost never exists.

From the point of view of the applications, conditions on $g(t)$ and $f(t)$ which are sufficient are usually more important than those which are necessary in order that (6) be of type $\left(^{*}\right)$. On the other hand, it is precisely a (sufficiently inclusive) necessary condition the formulation of which seems to lead to peculiar difficulties. In order to illustrate this, suppose that $g(t)$ vanishes identically, so that (6) reduces to

$$
x^{\prime \prime}+f(t) x=0 .
$$

Then the first of the conditions (5) is violated (at every $t$ ). Nevertheless, (7) can be of type $\left(^{*}\right)$. For suppose that $f(t)$ tends to $\infty$ as $t \rightarrow \infty$ (so that the second 
of the conditions (5) is very much satisfied) and that $f(t)$ is of a sufficiently regular but not too rapid growth; for instance, that $f(t)$ is a not too rapid logarithmico-exponential function satisfying $f(t) \rightarrow \infty$. Then it is known, $c f$. [10], that two, linearly independent, solutions of (7) are asymptotically proportional to the real and imaginary parts of

$$
[f(t)]^{-\frac{1}{2}} \exp \left\{i \int^{t}[f(s)]^{\frac{1}{2}} d s\right\} .
$$

Since $\cos \int^{t}$ and $\sin \int^{t}$ oscillate between finite bounds, it follows that (7) is of type $(*)$ if and only if $1 / f(t)$ is exponentially small (incidentally, the restriction to functions $f(t)$ of "regular growth" cannot be omitted here; $c f$. [11]).

5. Fortunately, the situation proves to be less intricate with regard to conditions which are sufficient in order that (6) be of type $\left(^{*}\right)$. Of course, it cannot suffice to assume only (5) (for all $t$ ). For instance, if

$$
g(t)=\frac{c_{1}}{t}, \quad f(t)=\frac{c_{2}}{t^{2}}, \quad(1 \leqq t<\infty),
$$

where $c_{1}$ and $c_{2}$ are positive constants, then (2) is satisfied. But no solution of (6) (except, of course, the trivial solution $x(t) \equiv 0$ ) is a (*)-function and so, in particular, the case (8) of (6) cannot be of type $\left(^{*}\right)$. In fact, $x(t)=t^{\lambda}$ is now a solution of (6) if $\lambda$ is either root of a quadratic equation

$$
\lambda^{2}+\left(c_{1}-1\right) \lambda+c_{2}=0 .
$$

It turns out that all that goes wrong in the example (8) is that the satisfaction of (5) is now tenuous, since $g$ and $f$, though positive for fixed $t$, fail to stay above positive bounds as $t \rightarrow \infty$. In fact, the following criterion will be proved:

(ii) If $g=g(t)$ and $f=f(t)$, where $t_{0} \leqq t<\infty$, are continuous functions which stay between positive bounds as $t \rightarrow \infty$, then all solutions $x(t)$ of (6) are exponentially small.

The assumptions of (ii) reduce to (5) in the stationary case. Hence it is clear that (ii) applies not only in the non-oscillatory case of a "decay." For instance, if $g(t)$ is a positive constant, which can be chosen to be 1 , then (6) reduces to

$$
x^{\prime \prime}+x^{\prime}+f(t) x=0,
$$

and the assumptions of (ii) require the existence of two positive constants $f_{0}$, $f^{0}$ satisfying $f_{0} \leqq f(t) \leqq f^{0}$ as $t \rightarrow \infty$. But StuRm's comparison theorem shows that (9) is oscillatory if the case $f(t) \equiv f_{0}$ of (9) is. Hence (9) is oscillatory whenever

$$
\lim _{t \rightarrow \infty} \inf f(t)>\frac{1}{4} .
$$

In fact, the case $f(t)=f_{0}$ of (9) has the solution $x(t)=e^{\lambda t}$ if $\lambda^{2}+\lambda+f_{0}=0$, and the roots of this quadratic equation are complex if $f_{0}>\frac{1}{4}$. 
The reason why the particular case (9) of (6) has been mentioned explicitly is that (ii) will be proved via its particular case $g(t) \equiv 1$ :

(ii bis) If $f(t)$, where $t_{0} \leqq t<\infty$, is a continuous function which stays between positive bounds as $t \rightarrow \infty$, then all solutions $x(t)$ of (9) are exponentially small.

In fact, the four theorems (i), (i*), (ii), (ii bis) will be proved by first establishing the following two implications: (ii bis) $\rightarrow$ (i) and (i) $\rightarrow$ (ii). Since (ii) $\rightarrow$ (ii bis) and (i*) $\rightarrow$ (i) are obvious, and since the substitution

$$
t=-\log r \text { or } r=e^{-t} \quad(t \rightarrow \infty, r \rightarrow+0)
$$

shows that (ii) $\rightarrow\left(i^{*}\right)$, it follows that all four theorems are equivalent and that, therefore, it will be sufficient to prove (ii bis).

Incidentally, an elaboration of the proof of (ii) would show that $g(t)$ and $f(t)$ can be allowed to come arbitrarily close to 0 , provided that this takes place sufficiently slowly (more specifically, so as to assure the convergence, as $t \rightarrow \infty$, of certain integrals involving the positive functions $g, f$ ).

6. Proof of (i) $\rightarrow$ (ii). If $t_{0}$ is large enough and $t_{0} \leqq t<\infty$, then, by the assumptions of (ii), there exist four constants, say $a, b, A, B$, satisfying

$\left(12_{1}\right) \quad f(t)>a>0, \quad g(t)>b>0 ; \quad\left(12_{2}\right) \quad f(t)<A, \quad g(t)<B$.

Put

$$
d r=-h d t, \text { where } h=h(t)=\exp \left\{-\int_{t_{0}}^{t} g(s) d s\right\},
$$

and replace $t$ by the independent variable $r$. Then, if $t=t_{0}$ corresponds to $r=r_{0}$, it is clear from (13) and from the second of the assumptions (12 $12_{1}$ that $t_{0}<t<\infty$ goes over into $r_{0}>r>0$ (with $r \rightarrow 0$ as $t \rightarrow \infty$ ), and that

$$
0<h<\text { const. } e^{-b t} \text {, hence } r<\text { const. } e^{-b t} \text {, }
$$

where both constants are positive. Similarly, if the second of the assumptions $\left(12_{2}\right)$ is used,

$$
h>\text { Const. } e^{-B t} \text {, hence } r>\text { Const. } e^{-B t} .
$$

Consequently, $t /(-\log r)$ stays between two positive bounds as $t \rightarrow \infty$ or $r \rightarrow 0$. Hence, a function $x$ of $t$ is exponentially small if and only if it satisfies (1) (with some constant $\alpha>0$ ) as a function of $r$.

It also follows from $\left(14_{1}\right)$ and $\left(14_{2}\right)$ that the assumptions imposed on $f$ by $\left(12_{1}\right)$ and $\left(12_{2}\right)$ imply that, if $\omega=\omega(r)$ is the positive function defined by

$$
\omega^{2}=\frac{f}{h^{2}} \text {, }
$$

then $r \omega(r)$ stays between two positive bounds as $r \rightarrow 0$. Accordingly, the assertions of (ii) for (6) are equivalent to the assertions of (i) for (2), if the con- 
nection between (2) and (6), where the primes denote $d / d t$, is defined by (13) and (15).

Finally, trivial substitutions show that (6) is identical with (2) by virtue of (13) and (15). Hence, if (i) is proved, then (ii) will follow.

7. Proof of (ii bis) $\rightarrow$ (i). The substitution (11) shows that (i) is equivalent to (ii bis). Hence, if (ii bis) is proved, then (i) (and so (ii) as well) will follow.

8. The "variation of constants" in (ii bis). Let $x=x_{1}(t)$ and $x=x_{2}(t)$ be two solutions of (9) which are real-valued and linearly independent. Then $x_{1}+i x_{2}=$ $z=z(t)$ is a complex-valued solution of (9), that is,

$$
z^{\prime \prime}+z^{\prime}+f(t) z=0
$$

and, since the Wronskian $x_{1} x_{2}^{\prime}-x_{2} x_{1}^{\prime}$ does not vanish at any $t$,

$$
z \neq 0 \quad\left(\text { and } z^{\prime} \neq 0\right) \text { for } t_{0} \leqq t<\infty .
$$

The assumption of (ii bis) is the existence of two constants satisfying

$$
c \leqq f(t) \leqq C \text {, where } c>0 .
$$

This assumption will be used for appropriate choices of the value of $\gamma=$ const. $>0$ in the trivial differential equation

$$
w^{\prime \prime}+w^{\prime}+\gamma w=0,
$$

with which the given differential equation (16) will now be "compared" (this refers, in a certain extent, to a "comparison" in Sturm's sense, even though the solution to be considered must be chosen as complex-valued in the oscillatory case).

First, $w=e^{-\mu t}$ is a solution of (19) if $\mu=\mu(\gamma)$ is either root of

$$
\mu^{2}-\mu+\gamma=0 .
$$

Since $e^{-\mu t} \neq 0$, it follows from (17) that the given $z=z(t)$ and either $\mu$ determine a unique $y=y(t)$ for which the relation

$$
z=y e^{-\mu t} \text {, where } y \neq 0 \text { for } t_{0} \leqq t<\infty,
$$

holds as an identity in $t$.

Suppose that the roots of (20) are complex, say $\mu=\alpha \pm i \beta$, where $\alpha$ and $\beta \neq 0$ are real, and consider, for instance, the root

$$
\mu=\alpha+i \beta, \text { where } \beta>0
$$

(the other root of (20) is $\mu=\alpha-i \beta$ if $\beta>0$, and $\mu=1-\alpha$ if $\beta=0$ ). A direct substitution shows that the "variation of constants" (21) transforms (16) into

$$
y^{\prime \prime}+(1-2 \mu) y^{\prime}+\left(\mu^{2}-\mu+f\right) y=0 .
$$


But (20) and (22) show that (23) can be reduced to

$$
y^{\prime \prime}-2 i \beta y^{\prime}+(f-\gamma) y=0 .
$$

Finally, if

$$
y=u+i v
$$

where $u$ and $v$ (and, by assumption, the function $f=f(t)$ and both constants $\beta, \gamma)$ are real, then it is clear that the complex differential equation (24) is equivalent to the following system of two real differential equations:

$$
u^{\prime \prime}+2 \beta v^{\prime}+(f-\gamma) u=0, \quad v^{\prime \prime}-2 \beta u^{\prime}+(f-\gamma) v=0 .
$$

Let this system be written in the vectorial form,

$$
s^{\prime \prime}-2 \beta J s^{\prime}+(f-\gamma) I s=0, \text { where } s=\left(\begin{array}{l}
u \\
v
\end{array}\right)
$$

and

$$
J=\left(\begin{array}{rr}
0 & -1 \\
1 & 0
\end{array}\right), \quad I=\left(\begin{array}{ll}
1 & 0 \\
0 & 1
\end{array}\right)
$$

9. Proof of (ii bis). Choose the $\gamma$, first occurring in (19), to be the $C$ of (18). Then $f(t)-\gamma \leqq 0$ throughout. Hence the coefficient of $s$ in (26) is a real, symmetric, non-positive definite matrix at every $t$, where $t_{0} \leqq t<\infty$. On the other hand, the coefficient of $s^{\prime}$ in (26) is a real, skew-symmetric, constant matrix. It follows therefore from the main result of [12] that (26) possesses two real, linearly independent (vector) solutions, say $s=s_{1}(t)$ and $s=s_{2}(t)$, having the property that, if $s=s(t)$ denotes either of them, then $|s(t)|^{2}$ is a non-increasing function for $t_{0} \leqq t<\infty$. In fact, an application of the device of the variation of constants shows that the occurrence of the constant skew-symmetric matrix $-2 \beta J$ in (26) has no bearing on the problem.

In particular, $|s(t)|^{2}=u^{2}+v^{2}$ tends to a finite, non-negative limit as $t \rightarrow \infty$. It follows therefore from (25), (21), and (22) (where $\alpha+i \beta$ can be replaced by $\alpha-i \beta)$ that (16) has two, linearly independent solutions $z(t)$ which are $O\left(e^{-\alpha t}\right)$ as $t \rightarrow \infty$. This means that all solutions $z(t)$ of (16) or, equivalently, all real solutions $x(t)$ of $(9)$, are $O\left(e^{-\alpha t}\right)$. Hence, all solutions of (9) are exponentially small, as claimed by (ii bis), if $\alpha>0$. But $\alpha>0$ is obvious. In fact, since $\gamma$ was chosen to be $C$, and since (18) implies that $C>0$, it is clear from (20) and (22) that $\alpha>0$.

10. Note that the existence of a constant $c>0$ satisfying the lower limitation of (18) was not actually used. All that was used is that the upper limitation, $f(t) \leqq C$, be satisfied by a constant $C>0$ having the property that roots of the case $\gamma=C$ of the quadratic equation (20) become complex (in fact, the common real part, $\alpha$, of the roots $\mu=\alpha \pm i \beta$ is then positive by necessity, 
since the coefficients of (20) present two variations of sign). Since the roots of (20) are complex if $\gamma>\frac{1}{4}$, it follows that, in the (formally) oscillatory case, the assertion of (ii bis) can be improved as follows:

(ii* bis) If $f(t)$, where $t_{0} \leqq t<\infty$, is a continuous function satisfying the unilateral restriction (10) (for instance, if $f(t) \rightarrow \infty$, then all solutions of (9) are exponentially small.

Since (ii bis) and (i) are equivalent by virtue of (11), it is clear that (i) has an extension which relates to (i) itself in the same way as (ii* bis) relates to (ii bis). A corresponding remark holds for (ii).

The preceding proof dealt only with that case of the assumptions, (18), of (ii bis) in which the roots of (20) become complex for $\gamma=C$. But if the roots of (20) are real ( $c f$. the second part of the parenthetical remark made after (22)), then the proof is the same as, though methodically simpler than, in the formally oscillatory case, considered above. In fact, the real equation (9) need not be replaced by the complex equation (16), where $z=x_{1}+i x_{2}$, and so the vector equation (26) becomes replaced by either of two scalar equations. The details are straightforward enough to be omitted here.

\section{REFERENCES}

[1] M. BôchER, "On regular singular points of linear differential equations of the second order ...," Transactions of the American Mathematical Society, 1 (1900), pp. 40-53.

[2] E. Borel, Leçons sur les séries divergentes, Paris, 1901.

[3] R. Fricke, Lehrbuch der Algebra, vol. 1, Braunschweig, 1924.

[4] R. Grommer, "Ganze transzendente Funktionen mit lauter reellen Nullstellen," Journal für die reine und angewandte Mathematik, 144 (1914), pp. 114-166.

[5] P. Hartman \& A. Wintner, "Oscillatory and non-oscillatory linear differential equations," American Journal of Mathematics, 71 (1949), pp. 627-649.

[6] A. Liapounoff, "Problème général de la stabilité du mouvement," Annales de Toulouse, 9, (1907), pp. 203-474.

[7] O. Perron, "Über Stabilität und asymptotisches Verhalten der Integrale von Differentialgleichungssystemen," Mathematische Zeitschrift, 29 (1929), pp. 129-160.

[8] —-, "Die Ordnungszahlen linearer Differentialgleichungssyteme, ibid., 31 (1930), pp. 748-766.

[9] A. Wintner, "Asymptotic integrations of the adiabatic oscillator," American Journal of Mathematics, 69 (1947), pp. 251-272; cf. "The adiabatic linear oscillator," ibid., 68 (1946), pp. 385-397.

[10] - "On the normalization of characteristic differentials in continuous spectra," Physical Review, 72 (1947), pp. 516-517.

[11] - "Stability and high frequency," Journal of Applied Physics, 18 (1947), pp. 941-942.

[12] — " "On linear repulsive forces," American Journal of Mathematics, 71 (1949), pp. 362-366.

[13] — "A criterion of oscillatory stability," Quarterly of Applied Mathematics, 7 (1949), pp. 115-117.

[14] - "On linear instability," ibid., 13 (1955), pp. 192-195.

The Johns Hopkins University

Baltimore, Maryland 